\title{
TELETRABAJO Y CAPITALISMO DE VIGILANCIA
}

\author{
Ximena Roncal Vattuone* \\ https://orcid.org/0000-0002-3409-3837
}

RECIBIDO: Octubre 2020 / ACEPTADO: Noviembre 2020 / PUBLICADO: Enero 2021

Como citar: Roncal Vattuone, Ximena. (2021). Teletrabajo y Capitalismo de Vigilancia. Telos: revista de Estudios Interdisciplinarios en Ciencias Sociales, 23 (1), Venezuela. (Pp. 177-192).

DOI: www.doi.org/10.36390/telos231.14

\section{RESUMEN}

El confinamiento generado por la pandemia Covid-19 en marzo de 2020, aceleró el cambio tecnológico, llevando a los gobiernos y a las empresas a definir medidas de distinto tipo, no solo para atender la emergencia sanitaria, sino para continuar con sus actividades durante el aislamiento. Entre las modificaciones organizativas de mayor trascendencia se encuentra el teletrabajo o trabajo en casa, vanagloriado por sus bondades tanto en la autonomía del trabajador, la flexibilidad de horarios como en un mayor equilibrio entre la vida laboral y la vida familiar. El objetivo de este artículo es analizar el teletrabajo y sus principales características en el marco de un capitalismo de vigilancia que implementa mecanismos de control digital exacerbando la vigilancia y el monitoreo de las actividades de los trabajadores. Se revisa a Shoshana Zuboff (2019a, 2019b), Byung Chul Han (2014; 2013) y Michael Foucault (2002) como soportes teóricos para comprender la versión del Capitalismo Digital que transformó los diversos espacios de relacionamiento fundamentalmente la relación capital/trabajo que acrecentó la individualización del trabajo, así como la incorporación de nuevos métodos de control y vigilancia. La metodología que se utiliza es cualitativa con base en la revisión documental y de análisis reflexivo en el que la inmersión del investigador es sensible en la interpretación del mundo social. Con base en el análisis se concluye que las empresas y los empleados digitales han modificado la manera en que trabajadores y empresas se relacionan, transformado el mundo laboral; y que en un escenario de virus y crisis civilizatoria, el ordenamiento de los teletrabajadores se agudiza con los software de monitoreo como métodos de control cada vez más eficaces y sutiles.

Palabras clave: Teletrabajo; capitalismo de vigilancia; software de monitoreo; vigilancia.

\footnotetext{
* Dra. en Economía Política del Desarrollo. Docente/Investigadora de la Benemérita Universidad Autónoma de Puebla. Correo electrónico: eximi@hotmail.com
} 


\title{
Telecommuting and Surveillance Capitalism
}

\begin{abstract}
The confinement generated by the Covid-19 pandemic in March 2020 accelerated technological change, leading governments and companies to define different types of measures, not only to attend to the health emergency, but also to continue with their activities during the isolation. Among the most important organizational changes is teleworking or working at home, boasted for its benefits both in the autonomy of the worker, flexible hours and a better balance between work and family life. The objective of this article is to analyze teleworking and its main characteristics within the framework of a surveillance capitalism that implements digital control mechanisms, exacerbating the surveillance and monitoring of workers' activities. Shoshana Zuboff (2019a, 2019b), Byung Chul Han (2014; 2013) and Michael Foucault (2002) are reviewed as theoretical supports to understand the version of Digital Capitalism that transformed the various spaces of relationship fundamentally the capital/work relationship that increased the individualization of labor, as well as the incorporation of new methods of control and surveillance. The methodology used is qualitative based on documentary review and reflective analysis in which the immersion of the researcher is sensitive in the interpretation of the social world. Based on the analysis, it is concluded that companies and digital employees have modified the way in which workers and companies relate to each other, transforming the labor world; and that in a scenario of virus and civilization crisis, the ordering of teleworkers is sharpened with monitoring software as increasingly effective and subtle control methods.
\end{abstract}

Keywords: Teleworking; surveillance capitalism; monitoring; surveillance software.

\section{Introducción}

La reconfiguración del poder mundial estimuló la flexibilización, la desregulación, la precarización del trabajo y la generalización de la ley del valor en la economía mundial como columna vertebral de la estrategia globalizadora (Sotelo, 2003). El conocimiento y el dato desplazaron el eje de la acumulación capitalista hasta constituirse en los insumos centrales del moderno proceso productivo, "nuevos modos de organización societal y nuevas formas de cultura y vida cotidiana" (Kellner, 1989, p. 78).

Esta configuración de la sociedad capitalista impuso adecuaciones acordes al nuevo esquema del capitalismo digital que desplegó decisiones políticas y económicas para atender las demandas de reproducción del capital, la modificación de las relaciones del poder económico y político en las relaciones sociales de producción, en la organización del trabajo y la intensidad de la productividad, importantes avances en el conocimiento científico y tecnológico, que demandaron un tipo de trabajador flexible y con mayores niveles de cualificación.

De esta manera se incluyeron como categorías socioeconómicas tanto al trabajo físico de la mano de obra como al trabajo intensivo en conocimientos con el propósito de incrementar y mejorar la fuerza laboral en un criterio de formación de capital humano, adecuando la producción de valor y de plusvalor a las condiciones cambiantes que se entretejían en la escala internacional (Sotelo, 2007).

Si bien el trabajo ha existido en todas las sociedades, independientemente de su forma histórica, es en el capitalismo donde éste se convierte en una mercancía y donde su valor se 
determina por el tiempo de trabajo para su producción y reproducción. En su acepción más básica, el trabajo se define como la transformación de un objeto a través de la actividad humana, la cual utiliza determinados medios de producción para la generación de un producto con valor de uso y, en ciertas condiciones, con valor de cambio (De la Garza, 2009).

Para Vincent (1996) "el trabajo es, simultáneamente, un envite vital y un envite social, particularmente para quienes son sus principales proveedores, a quienes proporciona en gran medida su identidad" (Vincent, 1996, en línea). En tanto que:

"El proceso de trabajo es mucho más que eso, es ante todo una relación dinámica entre un conjunto de fuerzas de trabajo condicionadas y un conjunto multiforme de capitales. Es un momento de la puesta en contacto entre la forma valor de la técnica y la forma valor de la actividad para proporcionar una forma valor a los productos (materiales o inmateriales). En este sentido, el proceso de trabajo es un proceso de transformación de valores en valores". (Vincent, 1996, en línea)

El trabajo, según Marx (1970), es "el conjunto de las condiciones físicas y espirituales que se dan en la corporeidad, en la personalidad viviente de un hombre y que éste pone en acción al producir valores de uso de cualquier clase" (p.176). Por lo tanto, su producción y reproducción implica necesariamente la reproducción de la propia persona y el mantenimiento de su actividad vital, refutando las doctrinas que niegan el papel del trabajo como esencia de la acumulación capital, a pesar que en los procesos productivos automatizados el capital constante adquiere una mayor relevancia.

La revolución tecnológica informacional no sólo ha introducido la utilización de recursos innovadores en las empresas y la expansión en el uso de internet, sino que ha generado modificaciones en los patrones laborales, en las formas organizativas empresariales y en la extracción de valor que implica la información personal de los teletrabajadores como condición de la desposesión digital.

El germen de la productividad gravita en la tecnología de la generación del conocimiento y el almacenamiento de datos, donde el conocimiento de las habilidades propias favorece una mayor productividad y las relaciones de trabajo se ven modificadas por la individualización y diversificación de las actividades (Castells, 1996).

Una de las transformaciones del trabajo promovidas por la digitalización es el teletrabajo, "una de las primeras formas de flexibilidad laboral" (BID, 2019, p. 20); y la oportunidad que tiene "el capitalismo contemporáneo de apropiarse de espacios y vivencias, en principio, relativamente ajenas al mercado" (Álvarez, 2019, p. 7).

El teletrabajo es una especie de fusión de lo laboral y lo doméstico, se sitúa en la dimensión virtual que exige a los trabajadores la recapacitación y el perfeccionamiento de sus habilidades digitales, dado el mayor dinamismo en la utilización de herramientas tecnológicas cada vez más sofisticadas que define los tiempos actuales: flexibilidad, individualismo, implosión controlada e incertidumbre. El Covid 19 impuso el teletrabajo de forma casi obligatoria. La Comisión Económica para América Latina (CEPAL) (2020), señala que 96\% de las organizaciones y/o empresas en América Latina implementaron el teletrabajo, en mayor o menor medida. 
"La nueva etapa de la flexibilización laboral inaugura el teletrabajo, cuya incubación es notoria en medio de la pandemia, donde los registros de los estragos y quiebra de muchas empresas son evidentes, pero a largo plazo, interpretando las anotaciones de un investigador de la Universidad de Cambridge, en el corto plazo veremos cambios en la naturaleza del trabajo y la socialidad humana con apariencias más cercanos a los nuevos y valientes mundos de la ciencia ficción que a la realidad anterior al coronavirus" (Salazar, 2020, p. 211).

Más allá de la conciencia, el capitalismo digital establece también nuevas conductas y percepciones, nuevas sensibilidades y sociabilidades que embriagan sin que los sujetos se percaten de sus consecuencias (Chul Han, 2014). La robotización, la digitalización, la inteligencia artificial y el blockchain reestructuraron no solo las ocupaciones y tareas de los trabajadores (BID, 2019), sino también las formas tradicionales de control.

Desde la perspectiva de Byung Chul Han (2014), una forma distinta de esclavitud para explotar al trabajador es la que surge con los aparatos digitales, los cuales favorecen la movilidad $y$, en este sentido, permiten que la actividad laboral se desempeñe en horarios diferentes y en cualquier espacio. En consecuencia, "La libertad de la movilidad se trueca en la coacción fatal de tener que trabajar en todas partes (...) cada uno lleva consigo de aquí para allá el puesto de trabajo como un campamento. Ya no podemos escapar del trabajo" (Chul Han, 2013, p. 24).

Si bien el teletrabajo no es una actividad nueva y existen referencias de su desenvolvimiento, sobre todo a través de la operación de las empresas transnacionales, desde marzo del 2020, la pandemia de Covid 19 no solo obligó la implementación de distanciamientos sociales, sino que la tecnología se convirtió en la salvaguarda para que las actividades económicas en el mundo no se detuvieran totalmente: "Entre el primer y segundo trimestre de 2020, el uso de soluciones de teletrabajo aumentó un 324\% y la educación en línea, más del 60\%" (CEPAL, 2020, p.1).

Con base en los planteamientos anteriores, para el desarrollo de este artículo se utilizó la revisión y análisis documental considerando los criterios señalados por Consuelo Hoyos (2008, p.7-34), quien afirma que la indagación sobre el fenómeno se realiza "mediante la revisión detallada y cuidadosa que se hace de los documentos que tratan dicho tema" de tal manera de "generar nuevas comprensiones" sobre el objeto de estudio "más allá de la simple descripción y explicación del fenómeno", fortaleciendo "el conocimiento crítico sobre el nivel de comprensión que de él se tiene".

El Corpus analizado a lo largo del texto ha sido el siguiente: Se recurrió a las aportaciones de Nilles (1988), Padilla (1999), Malo (2018), Drucker (1992) Jean-Marie Vincent (1996) además de instancias como la Organización Internacional del Trabajo (OIT), el Acuerdo Marco Europeo y las legislaciones de Colombia, Costa Rica y México para identificar las principales concepciones sobre el teletrabajo. Se desarrolló una revisión a Shoshana Zuboff (2019a, 2019b), Byung Chul Han (2014; 2013) y Michael Foucault (2002) como soportes teóricos así como a Roitman $(2018 ; 2020)$ y Salazar (2020), que posibilitaron generar nuevas comprensiones sobre el Teletrabajo dentro la lógica del Capitalismo Digital. Asimismo, se estudiaron dos informes uno de la Organización Internacional del Trabajo (2020) y el otro de la CEPAL (2020), los cuales dieron cuenta sobre los efectos de la pandemia en el trabajo y sus implicaciones para el teletrabajo. 
En el presente trabajo se examinan las distintas conceptualizaciones sobre el concepto de teletrabajo; después se analiza al capitalismo de vigilancia que configura los elementos para comprender el despliegue de los métodos de control y vigilancia utilizados en el teletrabajo; a continuación se describen dos software de monitoreo de las actividades de los teletrabajadores; finalmente, en las conclusiones se reflexiona sobre un futuro incierto que se agudiza con formas de control digital en el capitalismo de vigilancia.

\section{Sobre el concepto de Teletrabajo}

Según la Nota Panorama laboral en tiempos del Covid-19: Impactos en el mercado de trabajo y los ingresos América Latina y el Caribe (OIT, 2020), el mundo se encuentra frente a un rápido aumento de la destrucción de empleo. El Covid-19 ha llevado a una crisis sin precedentes y con repercusiones adversas al mundo laboral, fundamentalmente en tres aspectos: 1) Incremento del desempleo y del subempleo a escala mundial. De acuerdo con el organismo, la pérdida de empleo oscilaría entre 5,3 millones y 24,7 millones de personas tomando en consideración un referente de más de 180 millones de desempleados en 2019.

2) Un importante decremento de los niveles de producción y elevado peligro de despido de los trabajadores. Los efectos adversos en la demanda de mano de obra han llevado a ajustes importantes tanto en materia de reducción salarial como en los horarios de trabajo y en un incremento sustancial en el nivel de subempleo. Y 3) Aproximadamente $80 \%$ de la fuerza de trabajo mundial ha resultado afectada por la ralentización de las actividades económicas. La pérdida significativa en los ingresos y el incremento de la cantidad de trabajadores en condiciones de pobreza y extrema pobreza se hace más que inminente, con salarios por debajo de $\$ 3,20$ dólares diarios, en paridad del poder adquisitivo (OIT, 2020).

El documento afirma que "La retracción del nivel de actividad económica tendrá un impacto fuertemente negativo sobre el mercado de trabajo que se canalizará a través del efecto que tiene la dinámica productiva sobre los ingresos, las remuneraciones y la calidad de las ocupaciones" (p.5). Asimismo, destaca que la tasa de desocupación en ambas regiones es en conjunto de 13\%, en comparación con la de 2019 que fue de 8,1\% lo cual está generando "la mayor crisis económica que América Latina y el Caribe en su conjunto ha experimentado en toda su historia desde que hay registros estadísticos a inicios del siglo XX." (p.3).

De igual manera, la CEPAL (2020), señala que la tasa de desocupación regional se ubicará alrededor del $13,5 \%$ al finalizar el 2020 , lo que representa un incremento de $5,4 \%$ en relación del valor registrado en $2019(8,1 \%)$. Millones de trabajadores están expuestos a la pérdida de ingresos y al despido, lo que conlleva a que las condiciones de pobreza de la población se incrementen en 45,4 millones dato que representa aproximadamente $37,3 \%$ de la población Latinoamericana.

La agudización de las contradicciones de la crisis sistémica tiene como punto de inflexión a la pandemia Covid -19, la cual instala con más fuerza modalidades de trabajo flexibles, móviles y una reconfiguración de los entornos y las relaciones laborales con dispositivos para dominar la mente y los cuerpos. De esta forma, se garantiza el poder reforzado de los nuevos amos digitales que muestra un capitalismo de vigilancia que articula un sistema totalitario, donde la libertad y "nuestras navegaciones en red acaban con el control social del poder analógico ejercido por una clase social o una élite dominante transversal" (Roitman, 2018, en línea). 
El teletrabajo irrumpió como una de las estrategias laborales alternativas más importantes y efectivas para contener los contagios, como una oportunidad para la descentralización de las actividades empresariales y la intensificación de la competencia en la impronta de que las empresas se convertirán "en las empresas digitales que han querido ser" (Cole, 2020).

Igualmente, el teletrabajo en tiempos de pandemia ha significado para las empresas la posibilidad de robustecer su dominación y la presencia de nuevas modalidades de acumulación. El imperativo del "Big data" les ha brindado también las posibilidades de hacer pronósticos sobre el comportamiento de sus empleados,dice Chul Han, (2014), así "la utilización de métodos de vigilancia de la fuerza de trabajo, además de reproducir la subordinación, la intensificación e incremento de la productividad, controla las emociones y el desenvolvimiento de los empleados en un sistema de poder desigual" (p. 14).

Foucault (2002), hacía referencia a los métodos de vigilancia como "observatorios" de la multiplicidad humana cuando señalaba que:

"Al lado de la gran tecnología de los anteojos, de las lentes, de los haces luminosos, que forman cuerpo con la fundación de la física y de la cosmología nuevas, ha habido las pequeñas técnicas de las vigilancias múltiples y entrecruzadas, unas miradas que deben ver sin ser vistas; un arte oscuro de la luz y de lo visible ha preparado en sordina un saber nuevo sobre el hombre, a través de las técnicas para sojuzgarlo y de los procedimientos para utilizarlo" (p.158).

Para comprender la naturaleza del teletrabajo (telework) ${ }^{1}$, diversos autores y organismos internacionales ofrecen definiciones del concepto de teletrabajo:

Nilles (1988), considera que se trata de distintas formas que sustituyen los desplazamientos laborales por tecnologías de la información "cualquier forma de sustitución de desplazamientos relacionados con la actividad laboral por tecnologías de la información" (p.310). El hombre del teletrabajo, decía Peter Drucker (1992), "se va a encontrar que su materia prima será la información y que sus máquinas serán ordenadores y sus vías de comunicación serán las telecomunicaciones" (p. 57).

Para Antonio Padilla (1999), el teletrabajo consiste en desarrollar una actividad laboral en un espacio diferente al asignado por la empresa o institución "empleadora cliente para la que se trabaja; en él se realiza un empleo intensivo de las tecnologías de la información; y supone que el valor añadido que aporta el teletrabajador a la empresa está relacionado con el uso de esas tecnologías" (p.57).

En otra aportación, la Organización Internacional de Trabajo (OIT) (2016), afirma que "el trabajo a distancia y la utilización de nuevas tecnologías entrañan siempre cambios organizacionales, parece apropiado definir el teletrabajo como una forma de organización del trabajo con las siguientes características: a) el trabajo se realiza en un lugar distinto del establecimiento principal del empleador 0 de las plantas de producción, de manera que el trabajador no mantiene un contacto

\footnotetext{
${ }^{1}$ Son sinónimos de teletrabajo: remote working (trabajo a distancia), telecommuting (teledesplazamiento), networking (trabajo en red), home office (trabajo remoto desde casa) y flexible working (trabajo flexible).
} 
personal con los demás colegas de trabajo y b) las nuevas tecnologías hacen posible esta separación al facilitar la comunicación. Además, el teletrabajo puede realizarse (en línea) (con una conexión informática directa) o (fuera de línea), organizarse de manera individual o colectiva, constituir la totalidad 0 una parte de las tareas del trabajador, y ser ejecutado por trabajadores independientes 0 trabajadores asalariados (p. 3).

En Europa, en el Acuerdo Marco Europeo lo refieren como "una forma de organización y/o de realización del trabajo, utilizando las tecnologías de la información en el marco de un contrato o de una relación de trabajo" (p.3), pero trasladando las labores a espacios diferentes a los utilizados por la empresa o institución, en alusión a que "El teletrabajo es voluntario para el trabajador y el empresario afectados" (p. 2).

En Latinoamérica, particularmente en Colombia, el concepto de teletrabajo se define como: "Una forma de organización laboral, que consiste en el desempeño de actividades remuneradas 0 prestación de servicios a terceros utilizando como soporte las tecnologías de la información y comunicación -TIC- para el contacto entre el trabajador y la empresa, sin requerirse la presencia física del trabajador en un sitio especifico de trabajo" (Congreso de Colombia, 2008).

En Costa Rica, el teletrabajo se considera como:

"la prestación de servicios de carácter no presencial fuera de las instalaciones del patrono, en virtud de la cual un trabajador puede desarrollar su jornada laboral desde su propio domicilio, centro que se destine para tal fin o en trabajos de campo, mediante el uso de medios telemáticos" (Ministerio de Trabajo y Seguridad Social, 2015)

En México, "el teletrabajo tiene presencia en el país desde 1997, con su ingreso como parte del mercado de la manufactura de software, situación que se dio después de la firma del Tratado de Libre Comercio para América del Norte" (Garmendia, 2008, p. 15). En el capítulo Términos y Definiciones de la Norma Mexicana en Igualdad Laboral y No discriminación (Secretaría de Economía, 2015), se precisa el Teletrabajo como la "Actividad laboral que permite trabajar de manera no presencial utilizando medios informáticos en un lugar distinto a las instalaciones del centro de trabajo" (p.13).

En junio de 2019, el senado mexicano aprueba la modificación del artículo 311 de la Ley Federal del Trabajo (Cámara de Senadores, 2019), incorporando un nuevo capítulo sobre teletrabajo al que denomina como:

"La forma de organización laboral que consiste en el desempeño de actividades remuneradas, sin requerirse la presencia física del trabajador en un sitio específico y utilizando como soporte las tecnologías de la información y la comunicación para el contacto entre el trabajador y empleador" (Cámara de Senadores, 2019).

El dictamen por el que se reforma el artículo 311 establece que el empleador es el responsable de proporcionar al empleado las herramientas necesarias para el desempeño de sus actividades del teletrabajo, además de definir las medidas de productividad donde ambas 
partes deberían asumir derechos y responsabilidades en un marco de colaboración mutua. De acuerdo con las experiencias de personas teletrabajadoras, estas condiciones muestran una realidad distinta, pues dotar de las herramientas óptimas a los trabajadores es un principio que no está en correspondencia con el capital.

En los distintos conceptos señalados, se identifica que la práctica del teletrabajo alude formas de organización y realización del trabajo a distancia a través de la utilización de plataformas virtuales; así como la reubicación de los espacios del empleador a las instalaciones del hogar del trabajador. Esta situación ha implicado una alteración del contenido mismo del trabajo, profundizando la brecha digital, y en la estructura salarial a partir de la discriminación en relación con el lugar geográfico desde el que desarrollan sus actividades los trabajadores (Malo, 2018).

De acuerdo a datos de la CEPAL (2020), el teletrabajo ha incrementado de forma significativa el tráfico de Internet y la mediación digital. En el primer semestre del 2020, tanto la actividad virtual como el uso de aplicaciones tecnológicas relacionadas con el teletrabajo se incrementó a 324\%, en plataformas como Zoom (cuyas acciones se incrementaron en $75 \%$ ) 0 Google Meets. De acuerdo con el informe ¿Quién paga la Cuenta? Gravar la Riqueza Para enfrentar la crisis de la COVID-19 en América Latina y el Caribe (2020), de marzo a junio de este año, 73 multimillonarios en América Latina y el Caribe aumentaron sus fortunas en 48.200 millones de dólares.

En este mundo en transición a un Nuevo Orden Mundial, de versión digital del capitalismo, se han establecido distintas formas de dominación y neocolonización bajo la forma de una economía de mercado tecnoliberal en la que "todo dispositivo, toda técnica de dominación genera objetos de devoción que se introducen con el fin de someter. Materializan y estabilizan el dominio" (Chul Han, 2014, p.14); y donde el big-data, la informática y las tecnociencias implementadas en los procesos productivos y de trabajo se subsumen a las lógicas del capital.

Diversos autores han planteado que las bondades potenciales del teletrabajo radican en la posibilidad que tiene el trabajador de desarrollar competencias digitales, determinar el lugar y el tiempo dedicado a las responsabilidades laborales, así como optimizar su calidad de vida, y conciliar familia y trabajo. Al respecto, el BID (2020), señala que el teletrabajo ofrece ventajas para quienes "tienen compromisos familiares y personales que les impiden trabajar fuera del hogar en horario completo 0 definido. También permite mayor accesibilidad, ayudando a derribar las barreras estructurales que enfrentan las personas con discapacidad para acceder a un empleo" (p.57).

Sin embargo, no se puede perder de vista el hecho de que el capitalismo digital continúa siendo una forma de capitalismo, la era del capitalismo de vigilancia, "que descubre la psique como fuerza productiva" (Chul Han, 2014, p.12), cuya tendencia inherente es la sobreexplotación de la fuerza de trabajo en tanto sistema de poder fincado en la flexibilización de la tasa explotación y despersonalización de la relación laboral como condiciones para acrecentar la cuota de ganancia y profundizar en la relación de subsunción del trabajo al capital. Al respecto, Marx (1970) señalaba:

"La característica general de la subsunción formal sigue siendo la directa subordinación del proceso laboral -cualquiera que sea, tecnológicamente hablando, la forma en que se le lleve a cabo - al capital. Sobre esta base empero, se alza un modo de producción no solo tecnológicamente específico que metamorfosea la 
naturaleza real del proceso de trabajo y sus condiciones reales: el modo capitalista de producción. Tan solo cuando éste entra en escena se opera la subsunción real del trabajo en el capital" (p.87).

En estos tiempos de exponencial incertidumbre, el trabajo se hace más dependiente y se sujeta con mayor fuerza a los requerimientos del capital, en la medida en que las posibilidades de quedar sin empleo se amplían y se destruyen los valores de la sociedad laboral, por lo "que la inseguridad endémica será el rasgo distintivo que caracterice en el futuro el modo de vida de la mayoría de los humanos" (Beck, 2007, p. 12).

Esta alianza del modo de producción capitalista con los avances de la tecnociencia, la era del capitalismo de vigilancia, ha cambiado también las reglas del juego en todas las dimensiones. Vivimos en una virtualización de la vida humana y control social (Salazar, 2020) que ha transformado el mundo en un estado mental donde lee, controla y vende el pensamiento (Schirrmacher, 2014). La información se consigue de forma sibilina y sin violencia física para controlar el cuerpo y la mente (Roitman, 2020).

En el teletrabajo desaparece la frontera público y privado, cada paso del trabajador es espiado y la fuerza de trabajo se controla por el propio sistema tecnológico. "Se trata de una contrarrevolución que está transformando no el trabajo en una casa acogedora, sino nuestra casa acogedora en una tortura de trabajo" (Varela, 2020, en línea).

El teletrabajo no solo tiene rostro de precariedad que se refleja en la enorme carga laboral, largas jornadas y exigencias de sobreexplotación más allá de una jornada laboral, sino que somete a los sujetos a un sistema de control que vigila su productividad, sus movimientos, extingue la "línea divisoria entre trabajo concreto/trabajo abstracto" (Salazar, 2020, p.208), trasgrede la privacidad y los derechos laborales a través de programas de vigilancia telemática funcionales a las relaciones laborales del capitalismo de vigilancia.

\section{El Capitalismo de Vigilancia}

En líneas anteriores se afirma que el capitalismo digital es básicamente capitalismo de vigilancia, "el que manda en el medio digital y orienta nuestra trayectoria hacia el futuro" (Zuboff, 2019a, p.35), donde los amos digitales van a "fijar nuevos principios para regularizar, afinar y universalizar el arte de castigar" (Foucault, 2002, p.83), articulado a un nuevo orden mundial y "una nueva tecnología del poder de castigar". En el Siglo XVIII, Foucault (2002), ya sentenciaba un nuevo orden económico, se trata pues de Vigilar y Castigar, pero también establece distinciones y excluye.

"El capitalismo de la vigilancia es un actor nuevo en la historia: original y sui generis a la vez. Es único en su especie y diferente a todo lo demás: un nuevo planeta separado, que se rige por su propia física del tiempo y el espacio, sus días de sesenta y siete horas, sus cielos esmeraldas, sus sierras invertidas y su agua seca" (Zuboff, 2019a, p.31).

El capitalismo de vigilancia es el Nuevo Orden Económico, un orden de las finanzas y las tecnologías digitales como las fuerzas inescrupulosas impulsadas por nuevos imperativos económicos que pasan por alto las normas sociales y anulan los derechos elementales asociados a una especie social que aboga por la autonomía individual (Zuboff, 2019a). Para el 
capitalismo de vigilancia, la experiencia humana se convierte en la cuarta mercancía ficticia, la cual va a la par con la tecnología digital:

"Los actuales poseedores del capital de vigilancia han creado una cuarta mercancía ficticia, fruto de la expropiación de las experiencias humanas reales cuyos cuerpos, pensamientos y sentimientos son tan vírgenes e inocentes como lo eran las praderas y bosques de los que rebosaba la naturaleza antes de que el mercado los absorbiera. Siguiendo esta lógica, la experiencia humana se encuentra mercantilizada por el capitalismo de vigilancia para renacer en forma de "comportamientos". Estos, traducidos a datos, se colocan en la interminable cola que alimenta la máquina ideada para, a partir de ellos, realizar predicciones que se compran y se venden" (Zuboff, 2019b, en línea).

En el espíritu del capitalismo de vigilancia, la mercancía informacional, la experiencia humana es sometida a la dinámica mercantil, además de ser considerada un producto predictivo, un sujeto que anticipa lo que realizará, "ahora, pronto o después" (Zuboff, 2019a, p. 25) y se acompaña del cercamiento del procomún digital con mecanismos de coacción donde la desobediencia se penaliza. El valor creado es el resultado de la extracción libre y gratuita de valor.

Sin duda, la pandemia no ha sacado lo mejor del capitalismo de vigilancia en esta búsqueda de reacomodo del capital, más bien ha dejado al descubierto una vez más las miserias que lo sostienen como la exacerbada producción de beneficios al servicio de sus propios fines con dispositivos que adoptan formas distintas de manifestación de poder y los capacita en su condición de poderosos a imponer su voluntad, poder que adquiere incluso una forma permisiva al grado que el sujeto sometido no tiene conciencia de su sometimiento (Chul Han, 2014).

En el mundo del teletrabajo, las "cámaras de seguridad" que garantizan el rendimiento eficiente y la optimización del tiempo se instalan de forma invisible y a precios más módicos en los hogares. Es importante para las empresas saber qué es lo que hacen durante el día los trabajadores, segundo a segundo. Los softwares permiten visualizar concienzudamente los contenidos de las computadoras, analizar la productividad y capturar pantallas, por ejemplo, es un escrutinio minucioso donde los sensores invaden la privacidad de los sujetos y rastrean no solo las actividades laborales, sino que registran datos de acontecimientos personales que son almacenados y se constituyen en la primera dimensión de la extraordinaria renta digital.

En el Panóptico, Foucault (2002) hace referencia a los mecanismos de control utilizados a finales del siglo XVIII cuando se declaraba la peste en las ciudades. Estos mecanismos resaltan su vigencia en las prácticas de vigilancia que adopta el capitalismo digital en el teletrabajo mediante los diversos softwares de supervisión o monitorización de la productividad, el rendimiento y la eficiencia de los empleados:

"Este espacio cerrado, recortado, vigilado en todos sus puntos, en el que los individuos están insertos en un lugar fijo, en el que los menores movimientos se hallan controlados, en el que todos los acontecimientos están registrados, en el que un trabajo ininterrumpido de escritura une el centro y la periferia, en el que el poder se ejerce por entero, de acuerdo con una figura jerárquica continua, en el que cada individuo está constantemente localizado, examinado y distribuido entre los vivos, 
los enfermos y los muertos -todo esto constituye un modelo compacto del dispositivo disciplinario" (Foucault, 2002, p. 182).

En el siglo XXI, la máquina del capitalismo de vigilancia es el ordenador y su disco duro que nos lee y escudriña diariamente, "pero el aparato como tal es inocente: todo depende de quién lo maneja y con qué finalidad. Una vez expresado el egoísmo humano mediante una formula, como ya es el caso, permite calcular una sociedad entera" (Schirrmacher, 2014, p.12).

De la misma manera que en el teletrabajo, "el esquema panóptico, sin anularse ni perder ninguna de sus propiedades, está destinado a difundirse en el cuerpo social; su vocación es volverse en él una función generalizada" (Foucault, 2002, p.191). Los softwares de monitorización instalados en las casas incrementan la capacidad de vigilancia de las empresas, quienes realizan un seguimiento de las horas de trabajo, acceden, recopilan datos sobre el desenvolvimiento de los trabajadores y envían los reportes a los empleadores de manera automática, asegurando de esta manera el autodisciplinamiento. "Además con el trabajo flexibilizado aumenta la presión por disminuir los tiempos muertos y reducir al mínimo las existencias (...) se intenta aprovechar al máximo la capacidad muscular y cerebral de los trabajadores." (Katz, 2000, pp. 6-7).

Para garantizar y configurar los dispositivos de control, las empresas compran y utilizan distintos softwares que permiten monitorear las actividades de los teletrabajadores, que acortan la desconexión física de las empresas y sus empleados. Los desarrolladores de software de las importantes compañías del Big-data diseñan nuevas y sofisticadas tecnologías, los mecanismos tradicionales de vigilancia se sustituyen por "los panópticos digitales que vigilan y explotan lo social de forma despiada" (Chul Han, 2014, p.11).

Existen distintos tipos de software de monitoreo, los cuales proporcionan valiosa información sobre el trabajador en su puesto de trabajo. La mayoría registra las actividades laborales a través de la identificación de los programas que son utilizados, el tiempo que se destina en las navegaciones en la red, capturas de pantalla en forma aleatoria, etc. En niveles más extremos, los softwares pueden inspeccionar todo lo que el empleado teclea en su computadora, realizar revisiones aleatorias por medio de videollamadas, así como vigilancia a través de la cámara de la computadora, la cual debe estar abierta, negando al trabajador la posibilidad de identificar en qué momento es observado detrás de la pantalla.

\section{DeskTime Software de Monitoreo}

DeskTime es un software diseñado para monitorear y medir la productividad de las actividades que desarrollan los teletrabajadores, inicia su operación cuando es encendida la computadora hasta el momento en que se apaga. El programa le posibilita a la empresa determinar las características de las actividades de los trabajadores en tres niveles de semáforo: las actividades productivas (verde), las actividades improductivas (naranja) y las actividades neutrales (gris) (DeskTime, en línea).

El software maniobra mediante un rastreo de aplicaciones concentradas en 14 funciones para el seguimiento y vigilancia de los teletrabajadores: i) Seguimiento automático del tiempo; ii) Seguimiento del título del documento, iii) Cálculo de costos; iv) Calendario de ausencias; v) Seguimiento de proyectos; vi) Capturas de pantalla; vii) Seguimiento del tiempo sin conexión; viii) Temporizador pomodoro; ix) Contactos del equipo; x) Integración de terceros; 
xi) Seguimiento de URL y aplicaciones; xii) Reportes personalizados; xiii) Facturación y xiv) Programación de turnos (Figura 1) (DeskTime, en línea).

DeskTime le permite a la empresa llevar a cabo un seguimiento automático del uso del tiempo, documentando la cantidad de éste dedicado a cada una de las actividades, tareas y otros proyectos durante el día con lo cual es factible medir la productividad del trabajador. Los empleadores tienen la facultad de rastrear la utilización de internet precisando el sitio web, el programa y la aplicación que está utilizando el empleado. Asimismo, evalúa a través del semáforo si la actividad desarrollada es productiva o improductiva en función a las tareas asignadas, los sitios web visitados y las actividades desarrolladas (DeskTime, en línea).

El programa controla y elabora informes sobre las labores de los trabajadores, así como de la empresa, identifica los sitios web utilizados en el trabajo y/o el tiempo dedicado a tareas específicas con la ayuda de varios filtros que coadyuvan en el análisis de los datos exactos que el programa necesita. Por si fuera poco, la vigilancia se despliega también a los tiempos en que el trabajador está sin conexión. Es decir, el tiempo de horas de trabajo que pasa el trabajador fuera de la computadora (DeskTime, en línea).

Figura 1.

Software de Vigilancia para teletrabajadores

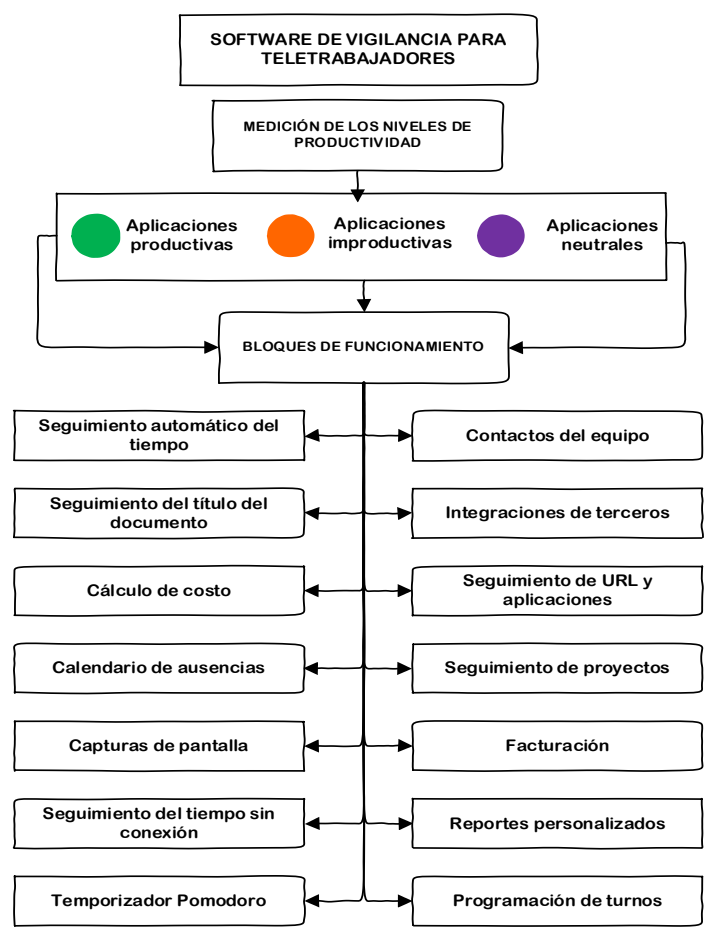

Fuente: Elaboración propia 
Las capturas de pantalla de la computadora se llevan a cabo con base en intervalos de tiempo aleatorios. De esta manera, los empleadores reciben informes sobre los programas y aplicaciones que son utilizados de manera activa. La integración de terceros es el proceso para sincronizar aplicaciones ocupadas en la empresa y optimizar los procesos. El capitalismo de vigilancia observa y registra cada movimiento que hace el trabajador en la red y reproduce en su totalidad la vida del teletrabajador (Chul Han, 2014).

\section{Kickidler Software de Monitoreo}

Kickidler es un software de seguimiento y monitoreo de computadoras en línea que permite evidenciar la ocupación de los trabajadores en tiempo real, los sitios web que visitan y las aplicaciones que utilizan. Es un programa que indica de forma automática las infracciones que se cometen en la jornada de trabajo, las cuales están en función de reglas predeterminadas. El funcionamiento de este software se lleva a cabo con base en tres componentes: un servidor que es el encargado de almacenar todos los datos recopilados por el programa; el programa Viewer, el cual se instala en el equipo desde donde se vigila, además de supervisar a los trabajadores tiene la función de analizar los reportes; el Grabber como dispositivo de captura del escritorio que es instalado en las computadoras de los vigilados (Kickidler, en línea).

Las funciones de este software son: i) Seguimiento automático del tiempo y estadísticas; ii) Monitoreo en línea de las computadoras; iii) Análisis de eficiencia; iv) Grabación de video de la pantalla; v) Control de ausencias; vi) Notificaciones automáticas; vii) Autocontrol; viii) Acceso remoto a la PC; xix) Registro de las teclas presionadas y $\mathrm{x}$ ) Control de sitios web visitados y aplicaciones ejecutadas (Figura 1) (Kickidler, en línea).

A diferencia de DeskTime, este software tiene mayores niveles de "perfección" en el ejercicio de la vigilancia. No solo rastrea el tiempo, sino que se complementa con informes estadísticos (gráficas y diagramas) que registran, entre otras cosas: los retrabajos y las "distracciones" del teletrabajador. Con el análisis de eficiencia, el software Kickidler (en línea) determina tanto el tiempo de trabajo como el tiempo personal e incluso cuando el trabajador se encuentra inactivo. La grabación de video de la pantalla le permite a la empresa fiscalizar todas las actividades de los empleados detrás de la computadora en cualquier intervalo de tiempo, las cuales se registran por los sistemas de información enviando notificaciones automáticas ante cualquier infracción.

El teletrabajador está solo, perfectamente individualizado y constantemente visible, ya no trabaja para sus necesidades, sino para el capital, en una nueva forma de subjetivización (Foucault, 2002; Chul Han, 2014). Por ello, en el monitoreo, la importancia del "Interfaz de Autocontrol" que le permite al teletrabajador ver sus estadísticas personales de productividad durante la jornada laboral, autoevaluarse y autovigilarse en su puesto de trabajo.

Aunque los procesos de vigilancia son de larga data, las características del teletrabajo y la revisión de los software de monitoreo nos han permitido acercarnos a los mecanismos de vigilancia y escrutinio de la conducta humana que se constituye en el activo intangible de la explotación que se ejerce hacia los teletrabajadores.

\section{Reflexiones finales}

Millones de personas están perdiendo sus trabajos como resultado de la pandemia y millones más se encuentran temerosos de perder su empleo pese a que en su mayoría tienen 
salarios precarios, los multimillonarios no tienen nada de qué preocuparse. Los rostros que encumbren los propósitos de bienestar y progreso están al descubierto.

El teletrabajo llegó para quedarse y representa dos peligros fundamentales que incrementan los costos para los trabajadores: primero, invisibiliza el trabajo colectivo y refuerza el individualismo de nuestra sociedad; segundo, las relaciones flexibles agudizan las situaciones de abuso laboral, haciendo invisible la tasa de explotación que incrementa la tasa de ganancia.

Con los nuevos dispositivos tecnológicos que facilitan el manejo de la psique y el control del cuerpo, el capitalismo de vigilancia post-pandemia agudizará con más fuerza las contradicciones capital-trabajo y será fuente de mayores desigualdades y de exclusión. Los dispositivos funcionales del capitalismo de vigilancia están modificando las relaciones sociales y laborales de las personas, se concentra fundamentalmente en una construcción de poder digital y en los principios de ordenamiento social cada vez más eficaces, sutiles y peligrosos.

Estamos asistiendo a una crisis civilizatoria, a la decadencia del sistema mundo moderno, una crisis de sentido de la vida, virus y pobreza. Sin duda, la realidad no brinda razones para el optimismo.

\section{Referencias Bibliográficas}

Álvarez Cantalapiedra, Santiago (2019). El capitalismo en la era digital. Revista Papeles de relaciones ecosociales y cambio global, 144, 2018/19, España. (pp. 5-10). Extraído de https://www.fuhem.es/wp-content/uploads/2019/05//ntroduccion-144.pdf

Beck, Ulrich (2007). Un nuevo mundo feliz. La precariedad del trabajo en la era de la globalización. Editorial Paidos Ibérica. México.

Banco Interamericano de Desarrollo (BID) (2019). El futuro del trabajo en América Latina y el

Caribe. Extraido de https://www.iadb.org/es/trabajo-y-pensiones/el-futuro-del-trabajoen-america-latina-y-el-caribe

Cámara de Senadores (2019). Proyecto de Decreto por el que se reforma el Artículo $311 \mathrm{y}$ Adiciona el Capítulo XII Bis, de la Ley Federal del Trabajo, en materia de Teletrabajo. Honorable Cámara de Senadores. México.

Castells, Manuel (1996) La era de la información. Economía, sociedad y cultura. Vol. 1. Siglo XXI. México

CEPAL (2020). Universalizar el acceso a las tecnologías digitales para enfrentar los efectos del COVID-19. Informe Especial. Naciones Unidas.

Cole, Matthew (2020). Why Silicon Valley Loves Coronavirus. Extraído de https://tribunemag.co.uk/2020/03/why-silicon-valley-loves-coronavirus

Congreso de Colombia (2008) Ley 1221. Diario Oficial 47.052. Colombia.

Chul Han, Byung (2013) En el Enjambre. Herder Editorial S.L. España.

Chul Han, Byung (2014) Psicopolítica. Neoliberalismo y nuevas técnicas de poder. Herder Editorial S.L. España.

De la Garza, Enrique (2009). Hacia un concepto ampliado de trabajo en Trabajo, empleo, calificaciones profesionales, relaciones de trabajo e identidades laborales. Vol. I. CLACSO. Argentina.

DeskTime Software de Monitoreo. Extraído de https://desktime.com/app/my Drucker, Peter (1992) La Gerencia. Editorial Ateneo, Argentina. 
Foucault, Michael (2002). Vigilar y castigar: nacimiento de la prisión.1a, edición. Siglo XXI Editores: Argentina.

Garmendia, Xóchitl (2008) Teletrabajo. Revista de Investigación Jurídica. México (pp.118).Extraído de https://www.tfja.gob.mx/investigaciones/historico/pdf/Teletrabajo.pdf

Hoyos, Consuelo (2008). Un modelo para la investigación documental. Guía teórico-práctica sobre construcción de Estados del Arte con importantes reflexiones sobre la investigación. Extraído de https://es.scribd.com/doc/16281901/UN-MODELO-PARAINVESTIGACION-DOCUMENTAL-29-04-08

Katz, Claudio (2000) La Teoría del Control Patronal: Balance de una discusión. Revista Estudios del Trabajo, (19). Argentina (pp. 3-34).Extraído de https://www.lahaine.org/katz/b2img/La\%20Teor\%C3\%ADa\%20del\%20Control\%20Patronal.pdf

Kellner, Douglas (1989) Postmodernism: Jameson Critique, Maisonneuve Press. California EUA.

Kickidler Software de Monitoreo. Extraído de https://www.kickidler.com/es/onlinemonitoring.html

Marx, Karl (1970). Manuscritos económicos filosóficos. Editorial Grijalbo: España.

Malo, Miguel (2018) Nuevas formas de empleo: del empleo atípico a las Plataformas Digitales.

Papeles de Economía Española. (156). España (pp.146-158). Extraído de http://grupo.us.es/iwpr/wp-content/uploads/2018/07/Del-trabajo-atipico-al-trabajo-enplataformas.pdf

Ministerio del Trabajo y Seguridad Social (2015) Decreto Ejecutivo N 39225-MP-MTSS-MICITT. La Gaceta 204. Costa Rica.

Nilles, Jack (1988) Traffic Reduction by Telecommuting: A Status Review and Selected Bibliography. Transportation Research A 22 (4), (pp.301-317). https://doi.org/10.1016/0191-2607(88)90008-8

Secretaría de Economía (2015) Norma Mexicana en Igualdad Laboral y No discriminación. Secretaría de Economía. México.

Organización Internacional del Trabajo (OIT) (2016). Las dificultades y oportunidades del teletrabajo para los trabajadores y empleadores en los sectores de servicios de tecnología de la información y las comunicaciones (TIC) y financieros. Extraído de http://www.ilo.org/wcmsp5/groups/public/---ed dialogue/---

sector/documents/publication/wcms_531116.pdf

Organización Internacional del Trabajo (OIT) (2020). Nota técnica "Panorama Laboral en tiempos de la COVID-19. Impactos en el mercado de trabajo y los ingresos en América Latina y el Caribe". Extraído de https://www.ilo.org/wcmsp5/groups/public/---americas/--ro-lima/documents/publication/wcms_749659.pdf

Padilla, Antonio (1999). Teletrabajo. Dirección y Organización. Ra-Ma, España.

Roitman, Marcos (2018). El control social de la mente en la era digital. Extraído de https://www.jornada.com.mx/2018/04/07/opinion/022a1mun

Roitman, Marcos (2020) La Post-Pandemia y el Capitalismo que viene. Extraído de https://www.jornada.com.mx/2020/06/14/opinion/014a2pol

Salazar, Robinson (2020). Pandemia y disciplinamiento social autoencierro con miedos y controles de obediencia. Revista Conjeturas Sociológicas, 8 (21), El Salvador. (Pp. 
199-218).

Extraído

de

http://revistas.ues.edu.sv/index.php/conjsociologicas/article/view/1534/1473

Schirrmacher, Frank (2014). Las trampas del juego capitalista. Ariel, España.

Sotelo, Adrián (2003) La reestructuración del mundo del trabajo, superexplotación y nuevos paradigmas de la organización del trabajo. Universidad Obrera de México. México.

Sotelo, Adrián (2007) La precarización del trabajo en la Década de 2000: Flexibilidad y Tensión Social. Memoria de Seminario Internacional: Las transformaciones del mundo del trabajo Bolivia, (pp. 12-16), Centro de Estudios para el Desarrollo Laboral y Agrario. Extraído de http://biblioteca.clacso.edu.ar/Bolivia/cedla/20190605032058/debatesocial_4.pdf

Varela, Raquel (2020) Teletrabajo "una contrarrevolución que convierte el hogar en tortura", afirma historiadora. Extraído de https://www.montevideo.com.uy/Ciencia-yTecnologia/Teletrabajo-una-contrarrevolucion-que-convierte-el-hogar-en-tortura--afirmahistoriadora-uc767430

Vincent, Jean-Marie (1996) La leyenda del trabajo. Extraído de http://www.mhh.domainepublic.net/ALGUNOSTEXTOS/SOCIOLOGIADELSALARIADO/ VINCENT/Vicent\%20La\%20leyenda\%20del\%20trabajo.pdf

Zuboff, Shoshana (2019a). La era del capitalismo de la vigilancia (Spanish Edition). Grupo Planeta/ Edición de Kindle. eBook

Zuboff, Shoshana (2019b). Un capitalismo de vigilancia. Extraído de https://mondiplo.com/uncapitalismo-de-vigilancia 